\title{
CT Imaging Correlates of Genomic Expression for Oral Cavity Squamous Cell Carcinoma
}

\author{
C.R. Pickering, K. Shah, S. Ahmed, A. Rao, M.J. Frederick, J. Zhang, A.K. Unruh, J. Wang, L.E. Ginsberg, A.J. Kumar,
} J.N. Myers, and J.D. Hamilton

tor

\begin{abstract}
BACKGROUND AND PURPOSE: Imaging correlates of genetic expression have been found for prognostic and predictive biomarkers of some malignant diseases, including breast and brain tumors. This study tests the hypothesis that imaging findings correlate with relevant genomic biomarkers in oral cavity squamous cell carcinoma.
\end{abstract}

MATERIALS AND METHODS: Surplus frozen tissue from 27 untreated patients with oral cavity squamous cell carcinoma who underwent preoperative CT imaging was analyzed for gene expression. A team of neuroradiologists blinded to the genomic analysis results reviewed an extensive list of CT findings. The imaging correlated with genomic expression for cyclin Dl, angiogenesis-related genes (vascular endothelial growth factor receptors and ligands), which relate to enhancement on the basis of other tumor types; and epidermal growth factor receptor, which may relate to proliferation and mass effect.

RESULTS: Expression of vascular endothelial growth factor receptors 1 and 2 correlated with the enhancement of the primary tumor $(P=$ .018 and $P=.025$, respectively), whereas the epidermal growth factor receptor correlated with mass effect $(P=.03)$. Other exploratory correlations included epidermal growth factor receptor to perineural invasion $(P=.05)$, and certain vascular endothelial growth factor receptors and ligands to mass effect $(P=.03)$ and increased $(P=.01)$ or decreased $(P=.02)$ primary tumor size.

CONCLUSIONS: We report that CT imaging correlates with gene expression in untreated oral cavity squamous cell carcinoma. Enhancement of the primary tumor and degree of mass effect correlate with relevant genomic biomarkers, which are also potential drug targets. Eventually, treatment decisions may be aided by combining imaging findings into meaningful phenotypes that relate directly to genomic biomarkers.

ABBREVIATIONS: ECS = extracapsular spread; EGFR = epidermal growth factor receptor; $\mathrm{HPV}=$ human papillomavirus; $\mathrm{HU}=$ Hounsfield unit; $\mathrm{OSCC}=$ oral cavity squamous cell carcinoma; VEGF = vascular endothelial growth factor

$\mathrm{H}_{\mathrm{c}}^{\mathrm{es}}$ ead and neck squamous cell carcinoma is the sixth most common cancer with approximately 600,000 cases per year worldwide and approximately 50,000 cases per year in the United States. ${ }^{1,2}$ Despite advances in treatment, the 5-year survival rate of patients with squamous cell carcinoma remains at $40 \%-50 \%{ }^{3}$ The most common site involved is the oral cavity. ${ }^{1}$ Known risk factors include alcohol; tobacco; and, to a lesser degree, human

\footnotetext{
Received July 6, 2012; accepted after revisions November 14.

From the Departments of Head and Neck Surgery (C.R.P., M.J.F., J.N.M.), Diagnostic Radiology (K.S., S.A., L.E.G., A.J.K., J.D.H.), and Bioinformatics and Computational Biology (A.R., J.Z., A.K.U., J.W.), The University of Texas MD Anderson Cancer Center, Houston, Texas.

Paper previously presented at: Annual Meeting of the American Society of Neuroradiology, April 21-26, 2012; New York, New York.

Please address correspondence to Jackson Hamilton, MD, MD Anderson Cancer Center, Unit 1482, PO Box 301402, Houston, TX 77230-1402; e-mail: jhamilton1@ mdanderson.org

-- Indicates open access to non-subscribers at www.ajnr.org

http://dx.doi.org/10.3174/ajnr.A3635
}

papillomavirus (HPV). ${ }^{4}$ As in most solid tumors, squamous cell carcinoma is usually caused by genetic alterations. Tumors in tobacco users tend to have more genomic alterations than in HPVassociated disease, ${ }^{5}$ and these alterations may contribute to treatment resistance.

Treatment failure most frequently takes the form of local and regional recurrences, but as disease control in these areas improves, treatment failures are more often a result of distant metastasis. The presence of cervical lymph node metastases, especially extracapsular spread (ECS), is a reliable adverse prognostic factor. ${ }^{6}$ The promise of basing treatment decisions on specific driver gene mutations or changes in expression, known as genomic biomarkers, has yet to be fully realized because of our poor understanding of the mechanisms of regional and distant metastases of squamous cell carcinoma.

Imaging phenotypes may convey relevant genomic information. Correlation of imaging morphologic features to the genotype and gene expression have already been made in other tumor 
sites, such as the breast, liver, or brain. ${ }^{7-15}$ Advantages of imaging include a larger area of coverage than pathologic specimens, which extends evaluation to tissue beyond the surgical resection margins, and less expense than combinations of multiple genomic tests, especially if obtained anyway as routine practice. If imaging findings can correlate with clinically useful molecular or genomic biomarkers, then a potential exists for improved tissue sample targeting, ${ }^{8}$ clinical decision making, response monitoring, ${ }^{16}$ and discovering and validating molecular targets. ${ }^{17}$ For example, higher expression of a gene correlated with invasion in glioblastoma by MR imaging and was subsequently found to be a prognostic biomarker of survival. ${ }^{17}$ However, imaging-to-genetic correlations have yet to be demonstrated for squamous cell carcinoma of the oral cavity (OSCC).

Epidermal growth factor receptor (EGFR) is a tyrosine kinase receptor on the cell surface related to cell growth that is both a predictive biomarker in head and neck squamous cell carcinoma ${ }^{18,19}$ and a chemotherapy target. ${ }^{3,20}$ EGFR expression has been related to the ratio of contrast-enhanced tumor and necrosis in brain tumors ${ }^{9}$ and primary tumor size and nodal metastasis for breast cancer. ${ }^{21}$ Vascular endothelial growth factor (VEGF) ligands and receptors are signaling proteins involved in angiogenesis and are targets for chemotherapy. ${ }^{9}$ VEGF expression has been related to brain and liver tumor enhancement and elevated perfusion. ${ }^{10,11,15}$ Cyclin D protein alters cell cycle progression and interacts with several proteins and receptors, including tumor necrosis factor alpha and retinoblastoma protein. Cyclin D1 may be a marker of poor prognosis and higher recurrence rates in OSCC, especially of the tongue, ${ }^{22,23}$ and has been associated with smaller, well-defined breast cancers. ${ }^{24}$ We tested the hypothesis that CT imaging findings correlate with selected genomic biomarkers in patients with OSCC. ${ }^{5,25}$

\section{MATERIALS AND METHODS \\ Genomics}

After institutional review board approval, untreated patients with OSCC underwent extensive genomic evaluation of surplus frozen tissue removed during a clinically indicated surgical procedure. A total of 27 of these patients had good-quality pathology specimens that underwent genomic analysis and also preoperative contrastenhanced CT examinations for retrospective review. Exome sequencing for some of these patients has been published previously. ${ }^{5}$ Only gene expression was examined for this analysis. Total RNA was isolated by using Tri Reagent (Sigma-Aldrich, St. Louis, Missouri) according to the manufacturer's instructions. RNA was hybridized to the Human Exon 1.0ST expression array platform (Affymetrix, Santa Clara, California) according to the manufacturer's instructions. Data were background-corrected with robust multiarray average and quantile normalized in R ( $\mathrm{R}$ statistical computing software; http://www.r-project.org/) with the aroma.affymetrix library. Probes were averaged across each gene. The groups of genes assessed in this preliminary study included cyclin D1 protein, EGFR, and VEGF ligands (VEGF-A, -B, and -C) and receptors including VEGF-r1 (also known as FMS-like tyrosine kinase receptor 1), VEGF r2 (kinase insert domain receptor or fetal liver kinase 1), and VEGF-r3 (FMS-like tyrosine kinase re-
Table 1: Patient demographics

\begin{tabular}{|c|c|c|c|c|}
\hline Patient No. & Age (y) & Sex & Stage & Subsite \\
\hline 1 & 62 & $M$ & $\mathrm{~T} 2 \mathrm{NO}$ & OT \\
\hline 2 & 66 & $M$ & T3 N2 & OT \\
\hline 3 & 62 & M & T3 No & OT \\
\hline 4 & 75 & $\mathrm{~F}$ & $\mathrm{~T} 4 \mathrm{~N} 2 \mathrm{C}$ & OT/FOM \\
\hline 5 & 23 & $M$ & $\mathrm{~T} 3 \mathrm{~N} 2 \mathrm{~b}$ & OT \\
\hline 6 & 40 & M & $\mathrm{T} 3 \mathrm{~N} 1$ & OT \\
\hline 7 & 50 & $M$ & $\mathrm{~T} 4 \mathrm{~N} 2 \mathrm{~b}$ & OT \\
\hline 8 & 69 & $M$ & $\mathrm{~T} 4 \mathrm{~N} 2 \mathrm{c}$ & OT/FOM \\
\hline 9 & 61 & $M$ & $\mathrm{~T} 2 \mathrm{~N} 1$ & OT \\
\hline 10 & 62 & $\mathrm{~F}$ & T3 No & OT \\
\hline 11 & 60 & $\mathrm{~F}$ & $\mathrm{~T} 2 \mathrm{~N} 2 \mathrm{~b}$ & ОT/BOT \\
\hline 12 & 40 & $M$ & T4 N2c & OT \\
\hline 13 & 60 & $M$ & T4 No & OT/FOM \\
\hline 14 & 50 & $M$ & T4a N2c & OT \\
\hline 15 & 85 & $\mathrm{~F}$ & $\mathrm{~T} 2 \mathrm{NO}$ & OT \\
\hline 16 & 56 & M & $\mathrm{T} 4 \mathrm{~N} 2 \mathrm{C}$ & FOM \\
\hline 17 & 50 & $\mathrm{~F}$ & $\mathrm{~T} 2 \mathrm{NO}$ & OT \\
\hline 18 & 74 & $M$ & T4 N2b & OT \\
\hline 19 & 53 & $M$ & T3 No & OT \\
\hline 20 & 61 & $\mathrm{~F}$ & $\mathrm{~T} 2 \mathrm{~N} 2 \mathrm{~b}$ & OT \\
\hline 21 & 76 & $\mathrm{~F}$ & $\mathrm{~T} 2 \mathrm{NO}$ & OT \\
\hline 22 & 56 & $M$ & $\mathrm{~T} 3 \mathrm{~N} 2 \mathrm{~b}$ & Bucca \\
\hline 23 & 48 & $M$ & $\mathrm{~T} 2 \mathrm{~N} 1$ & FOM \\
\hline 24 & 74 & $M$ & T4 No & Gingiva \\
\hline 25 & 64 & $M$ & $\mathrm{~T} 2 \mathrm{~N} 1$ & FOM \\
\hline 26 & 62 & $M$ & TINO & Bucca \\
\hline 27 & 67 & $M$ & $\mathrm{~T} 4 \mathrm{~N} 2 \mathrm{c}$ & FOM \\
\hline
\end{tabular}

Note:-BOT indicates base of tongue; F, female; FOM, floor of mouth; M, male; N, nodal stage; OT, oral tongue; T, tumor stage.

ceptor 4). Additional demographic, pathology, and follow-up data were gathered. HPV status was noted if available.

\section{Imaging}

Imaging analysis was done by consensus of 2 board-certified neuroradiologists. A third reader was used to resolve discrepancies. All readers were blinded to genetic results.

The CT technique changed during the course of the study. Most patients underwent imaging on a 16-detector LightSpeed CT scanner (GE Healthcare, Milwaukee, Wisconsin) with axial 1.25 - to $2.5-\mathrm{mm}$ collimation at $120 \mathrm{kVp}$ and $200-240 \mathrm{~mA}$, after injection of $120 \mathrm{~mL}$ of Omniscan (GE Healthcare) at $3 \mathrm{~mL} / \mathrm{s}$ with a 90-second delay. A second angled acquisition was performed as necessary to eliminate dental artifacts.

Bidimensional measurements of the primary tumor and, if present, the largest metastatic lymph node were made on axial images. The cross-sectional area [ for an ellipse $=\pi(1 / 2$ long axis $\times$ $1 / 2$ short axis)] and the ratio of the long axis over the short axis were calculated. Enhancement of the primary site and of the largest solid component of lymph nodes was measured by a representative region of interest in Hounsfield units (HU). The enhancement of the submandibular gland was used as a positive control to normalize the values among patients (lesion HU/submandibular gland $\mathrm{HU}$ ). For the primary tumor, borders were judged as ill- vs well-defined and as regular vs irregular. Invasion into adjacent structures was noted. Mass effect was graded as none, mild if there was $<1 \mathrm{~cm}$ displacement of airway or other adjacent structures, moderate for $1-2 \mathrm{~cm}$ displacement and marked if $>2 \mathrm{~cm}$. Perineural extension of the primary tumor or ECS from lymph nodes was assessed both by imaging and subsequent pathologic 
examination. Necrosis was estimated by fifths (0\%-20\%, 20\%$40 \%$, etc) for both primary and lymph nodes. The presence of calcification or ulceration was also noted.

\section{Statistics}

Associations between gene expression and imaging variables were assessed by use of Pearson correlation and ordinal regression for contiguous and categoric imaging features, respectively. Bonferroni correction set the adjusted significant level at a $P$ value $\leq$

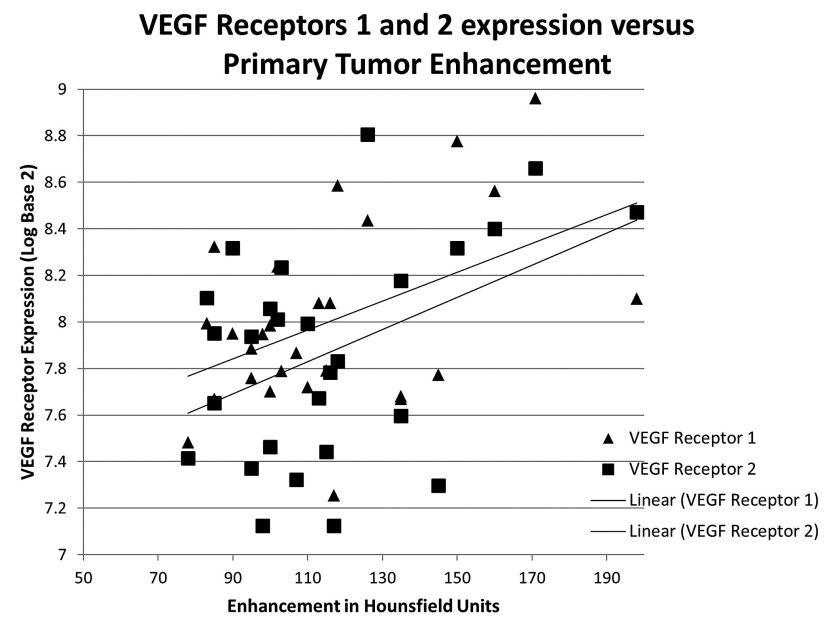

FIG 1. Scatterplot showing relationship of increased enhancement and VEGF receptors 1 and 2 expression. The linear fitting demonstrates that as the absolute enhancement in $\mathrm{HU}$ increases, the receptor expression (in log base 2) also increases.

\section{Graded Mass Effect versus EGFR Expression}

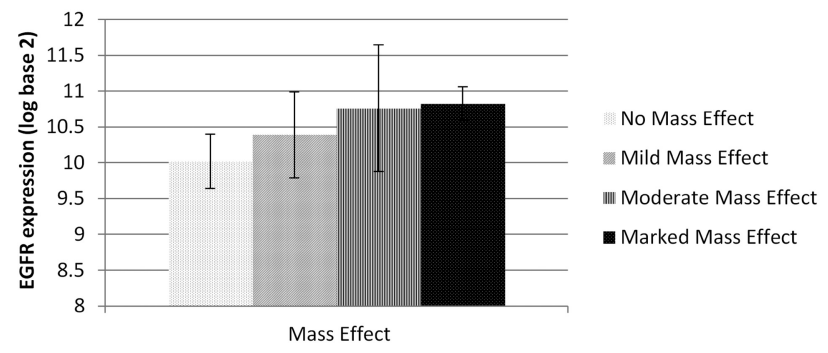

FIG 2. Bar graph reflecting positive correlation between the grading of mass effect and the expression of EGFR (in log base 2) with standard deviation bars.

Table 2: Correlations of gene expression to maximal axial cross-sectional area

\begin{tabular}{lllc}
\hline \multicolumn{1}{c}{ Imaging Finding } & Gene Expressed & $\begin{array}{c}\text { Coefficient } \\
\text { Value }\end{array}$ & $\begin{array}{c}\text { Significance } \\
(\boldsymbol{P} \text { Value })\end{array}$ \\
\hline Mass effect & EGFR & OR: +1.2 & $.032^{\mathrm{a}}$ \\
Perineural invasion & EGFR & OR: +3.1 & .047 \\
Axial cross-sectional area $\left(\mathrm{cm}^{2}\right)$ & VEGF ligand A & PC: +0.49 & .014 \\
Axial orthogonal distance $(\mathrm{cm})$ & VEGF ligand A & PC: +0.47 & .010 \\
Mass effect & VEGF ligand B & OR: +2.8 & .026 \\
Enhancement of primary tumor (HU) & VEGF receptor 1 & PC: +0.45 & $.018^{\text {a }}$ \\
Axial maximal diameter (cm) & VEGF receptor 2 & PC: -0.44 & .023 \\
Axial cross-sectional area (cm $\left.{ }^{2}\right)$ & VEGF receptor 2 & PC: -0.42 & .029 \\
Enhancement of primary tumor (HU) & VEGF receptor 2 & PC: +0.43 & $.025^{\text {a }}$ \\
Enhancement of submandibular gland (HU) & VEGF receptor 2 & PC: +0.43 & .026 \\
\hline
\end{tabular}

Note:- $\mathrm{cm}$ indicates centimeter; OR, ordinal regression; PC, Pearson correlation.

${ }^{a}$ Denotes confirmation of a priori hypothesis.
.00036 for an unadjusted $P$ value $\leq .05$. Specific hypotheses to be tested based on other tumor types included 1) positive correlations between VEGF expression and enhancement ${ }^{10,11}$; 2) EGFR expression with necrosis, tumor size, and presence of nodal metastasis $^{9,21}$; and 3) a negative correlation with cyclin D1 expression and tumor size and invasion. ${ }^{24} \mathrm{We}$ also hypothesized that because EGFR relates to growth and proliferation, it would have a positive correlation to mass effect, ${ }^{8}$ though this trend has not been reported previously. To check for confounding factors and groups of imaging features, we also assessed the associations among imaging features themselves by using ordinal regression (categoric vs continuous), Pearson correlation (continuous vs continuous), or the Fisher exact test (categoric vs categoric).

\section{RESULTS}

Images were available from 2001-2010. The average age of the patients was $59 \pm 13$ years; of 27 patients, 20 (74\%) were men (Table 1). Most tumors were located in the oral tongue (19/27, or $59 \%$ ), with 4 tumors involving the floor of the mouth, 3 tumors involving the bucca or gingiva, and 4 tumors involving a combination of sites. Primary tumors were staged pathologically as T1 in 1 patient, T2 in 9 patients, T3 in 6 patients, and T4 in 11 patients. Nodal staging was N0 in 10 patients, N1 in 4 patients, N2 in 13 patients, and N3 in no patients. Only 1 patient with a T4 N2b cancer of the oral tongue was found to test positive for HPV.

Imaging results revealed a spectrum of findings. The average size of the primary tumor was $3.6 \pm 1.2 \mathrm{~cm}$ in the long axis with an elliptic cross-sectional area of $6.1 \pm 5.8 \mathrm{~cm}^{2}$. All primary tumors enhanced to some degree with either avid enhancement (absolute $119 \pm 29 \mathrm{HU}$ or normalized ratio $1.2 \pm 0.3)$, minimal enhancement ( 3 patients: absolute $89 \pm 16 \mathrm{HU}$ or normalized ratio $1.2 \pm$ 0.2 ), or in 1 case rim enhancement with a primarily necrotic tumor. A total of 9 patients had invasion deep into the muscle, 3 had invasion into the bone, and the remaining patients did not have deep invasion. Although no gross perineural extension was seen on imaging, 8 patients had perineural invasion by pathologic examination. Most tumors were irregular $(n=22)$, ill defined $(n=$ $21)$, and partially necrotic $(n=21)$, and had mild to moderate mass effect $(n=19)$ with few ulcers $(n=9)$ and no calcifications. Of the 17 patients with pathologic nodal disease, 2 had tumors that were not readily detected on CT scanning. Of the affected largest lymph nodes, the average size was $1.4 \pm 0.4 \mathrm{~cm}$ in the long axis. Most lymph nodes enhanced $(n=$ 12) and were necrotic $(n=10)$, with 8 patients having ECS on pathologic examination (only one of which was identified on imaging). In these presurgical patients, no nodal calcification was seen and distant metastatic disease was not found.

Expression of VEGF receptors 1 and 2 correlated with enhancement of the primary tumor $(P=.018$ and $P=.025$, respectively; Fig 1), whereas EGFR correlated with mass effect $(P=.03$; Fig 2$)$. The hypothesis that EGFR would corre- 
late with necrosis, primary tumor size, or lymph node metastases was not supported. No significant correlations were found for cyclin D protein, VEGF ligand C, or VEGF receptor 3. However, a trend was observed toward cyclin D1 expression and decreased orthogonal primary tumor size $(P=.11)$. Additional exploratory correlations that did not survive correction of significance values for multiple correlations are given in Table 2. As demonstrated in Fig 3, when the estimated cross-sectional area of the primary tumor increased, the expression of VEGF ligand A increased ( $P=$ $.01)$ but expression of VEGF receptor 2 decreased $(P=.03)$.

We also tested associations among imaging characteristics to look for confounding variables and groups of imaging characteristics. Larger tumors (maximal diameter, orthogonal, and crosssectional area) tended to have more mass effect $(P=.0018-P=$ .0035). The largest-diameter tumors correlated with invasion into muscle or bone $(P=.04)$. The patients who had moderate to marked mass effect or perineural invasion on pathologic examination had higher EGFR expression compared with those patients with neither moderate to marked mass effect nor perineural invasion ( $10.7 \pm 0.7$ vs $10.2 \pm 0.4$, respectively; $P=.05$ by unpaired $t$ test). Furthermore, $4(50 \%)$ of 8 patients with moderate to marked mass effect had perineural invasion, but 4 (33\%) of 12 patients with mild mass effect and none $(0 \%)$ of 7 patients with no

VEGF Ligand $A$ and Receptors 2 expression versus primary tumor cross sectional area

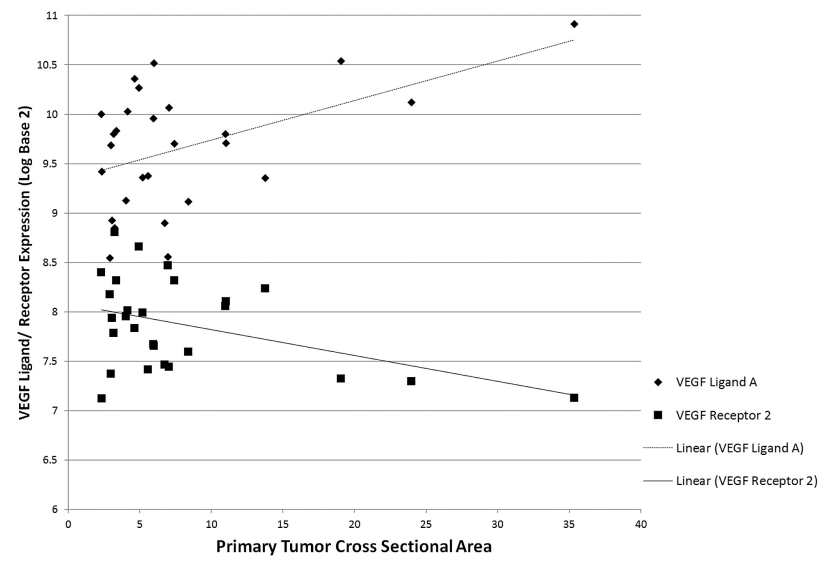

FIG 3. Scatterplot showing relationship of increasing primary tumor ellipsoid estimated cross-sectional area with increasing VEGF ligand $A$ and decreased receptor 2 expression (in log base 2) with linear fitting.
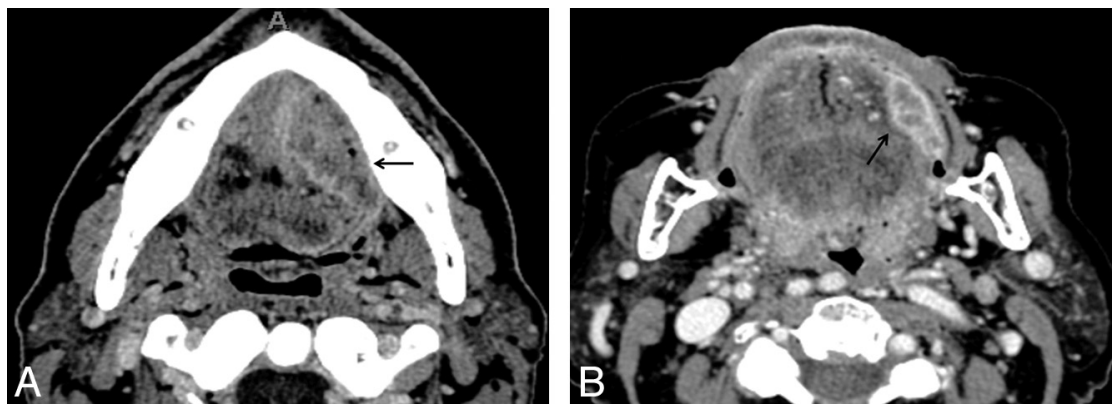

FIG 4. Example cases of highest expression for specific genes by use of contrast-enhanced axial CT images in different patients. A, Large OSCC located in the left tongue and at the floor of the mouth OSCC (arrow) with intrinsic tongue and perineural invasion (on histologic examination) and mass effect narrowing the airway. This tumor has the highest EGFR expression. B, Small left buccal tumor with avid enhancement with the highest VEGF receptor 1 and 2 expression. mass effect had perineural invasion. This trend was not quite statistically significant $(P=.08$; Fisher exact test between markedmass-effect group and no-mass-effect group). Also, enhancement tended to correlate with both the tumor and the submandibular gland as a regional control $(P=.00005)$, possibly because of contrast bolus differences among patients.

\section{DISCUSSION}

We tested correlates of the CT imaging appearance with genomic expression in a group of patients with previously untreated OSCC. Initial results suggest that degree of enhancement and mass effect indicate particular genetic alterations, similar to associations in other tumor types and related to drug targets. Limitations to this study included the small population, small number of genes tested, the relatively long period during which patients underwent imaging with varying imaging techniques, and the general nature of the retrospective review. None of the image-to-gene associations survived correction for the multiple correlations and, therefore, must be viewed with some skepticism. However, the associations demonstrated by other tumor types (eg, enhancement and VEGF receptors 1 and 2) are more likely to be true according to Bayesian probability. These simple correlations between a single imaging measurement and single gene expression are reductions of the complex interactions between the host stroma and the tumor.

Despite these limitations, the correlations suggest that grouping imaging findings into imaging phenotypes may further correlate with specific driver genes. Increased EGFR has correlated previously with worse stage and prognosis with treatment resistance. ${ }^{18,19,26}$ The association between size with mass effect and invasion, which also correlated with EGFR expression, may help to elucidate the association with tumor grade. The grouping of clinical and imaging findings into representative phenotypes could not only help in understanding and predicting genomic expression but also reinforce imagers' search patterns and the risk for invasion, which may not be directly visualized on imaging.

Smaller tumors had increased expression of different genes (VEGF receptor 2) than larger tumors (EGFR, VEGF ligand A), suggesting that tumor size and growth pattern are governed by underlying genomic expression and not solely by the time until diagnosis (Fig 3). Thus, a large tumor with invasion could suggest EGFR predominant expression, whereas a small tumor with avid enhancement could suggest increased VEGF receptor 2 expression (Fig 4). The differential expression of VEGF ligands and receptors with varying imaging features indicates that further work is needed to understand the reported association between high VEGF expression and increased recurrence rates with poor survival outcome, which did not clarify the subtypes of the receptor or ligand. ${ }^{27}$

\section{CONCLUSIONS}

In summary, we demonstrated correlations between CT findings and OSCC 
gene expression. The size and invasion of the tumor (and therefore stage and prognosis) may relate to specific driver gene expression. Further studies are needed with a greater number of patients, additional imaging modalities, additional tumor sites, and other potentially relevant genes to determine if grouping imaging features related to gene expression into imaging phenotypes is useful in the management of head and neck squamous cell carcinoma.

Disclosures: Curtis Pickering—RELATED: Grant: NIH and CPRIT.* Mitchell Frederick-RELATED: Grant: NIH and Texas CPRIT, Comments: Genomic studies were funded by peer-reviewed grants from the NIH and Texas Cancer Prevention Institute. Jiexin Zhang—RELATED: Grant: NIH and CPRIT.* *Money paid to institution.

\section{REFERENCES}

1. Ferlay J, Shin HR, Bray F, et al. Estimates of worldwide burden of cancer in 2008: GLOBOCAN 2008. Int J Cancer 2010;127:2893-917

2. Jemal A, Siegel R, Xu J, et al. Cancer statistics, 2010. CA Cancer J Clin 2010;60:277-300

3. Leemans CR, Braakhuis BJ, Brakenhoff RH. The molecular biology of head and neck cancer. Nat Rev Cancer 2011;11:9-22

4. Argiris A, Karamouzis MV, Raben D, et al. Head and neck cancer. Lancet 2008;371:1695-709

5. Agrawal N, Frederick MJ, Pickering CR, et al. Exome sequencing of head and neck squamous cell carcinoma reveals inactivating mutations in NOTCH1. Science 2011;333:1154-57

6. Spector ME, Gallagher KK, Light E, et al. Matted nodes: poor prognostic marker in oropharyngeal squamous cell carcinoma independent of HPV and EGFR status. Head Neck 2012;34:1727-33

7. Makkat S, Luypaert R, Stadnik T, et al. Deconvolution-based dynamic contrast-enhanced MR imaging of breast tumors: correlation of tumor blood flow with human epidermal growth factor receptor 2 status and clinicopathologic findings-preliminary results. Radiology 2008;249:471-82

8. Diehn M, Nardini C, Wang DS, et al. Identification of noninvasive imaging surrogates for brain tumor gene-expression modules. Proc Natl Acad Sci U S A 2008;105:5213-18

9. Furnari FB, Fenton T, Bachoo RM, et al. Malignant astrocytic glioma: genetics, biology, and paths to treatment. Genes Dev 2007;21:2683-710

10. Leo C, Giaccia AJ, Denko NC. The hypoxic tumor microenvironment and gene expression. Semin Radiat Oncol 2004;14:207-14

11. Maia AC, Jr., Malheiros SM, da Rocha AJ, et al. MR cerebral blood volume maps correlated with vascular endothelial growth factor expression and tumor grade in nonenhancing gliomas. AJNR Am J Neuroradiol 2005;26:777-83

12. Shimizu H, Kumabe T, Shirane R, et al. Correlation between choline level measured by proton MR spectroscopy and Ki-67 labeling index in gliomas. AJNR Am J Neuroradiol 2000;21:659-65

13. Emblem KE, Scheie D, Due-Tonnessen P, et al. Histogram analysis of
MR imaging-derived cerebral blood volume maps: combined glioma grading and identification of low-grade oligodendroglial subtypes. AJNR Am J Neuroradiol 2008;29:1664-70

14. Jenkinson MD, du Plessis DG, Smith TS, et al. Histological growth patterns and genotype in oligodendroglial tumours: correlation with MRI features. Brain 2006;129:1884-91

15. Kanematsu M, Osada S, Amaoka N, et al. Expression of vascular endothelial growth factor in hepatocellular carcinoma and the surrounding liver: correlation with angiographically assisted CT. AJR Am J Roentgenol 2004;183:1585-93

16. Emblem KE, Bjornerud A, Mouridsen K, et al. T(1)- and $\mathrm{T}(2)\left(^{*}\right)$ dominant extravasation correction in DSC-MRI: part II-predicting patient outcome after a single dose of cediranib in recurrent glioblastoma patients. J Cereb Blood Flow Metab 2011;31:2054-64

17. Zinn PO, Mahajan B, Sathyan P, et al. Radiogenomic mapping of edema/cellular invasion MRI-phenotypes in glioblastoma multiforme. PLoS One 2011;6:e25451

18. Rubin Grandis J, Melhem MF, Gooding WE, et al. Levels of TGFalpha and EGFR protein in head and neck squamous cell carcinoma and patient survival. J Natl Cancer Inst 1998;90:824-32

19. Pectasides E, Rampias T, Kountourakis P, et al. Comparative prognostic value of epidermal growth factor quantitative protein expression compared with FISH for head and neck squamous cell carcinoma. Clin Cancer Res 2011;17:2947-54

20. Bauman JE, Michel LS, Chung CH. New promising molecular targets in head and neck squamous cell carcinoma. Curr Opin Oncol 2012;24:235-42

21. Choi BB, Kim SH, Kang BJ, et al. Diffusion-weighted imaging and FDG PET/CT: predicting the prognoses with apparent diffusion coefficient values and maximum standardized uptake values in patients with invasive ductal carcinoma. World J Surg Oncol 2012;10:126

22. Mahdey HM, Ramanathan A, Ismail SM, et al. Cyclin D1 amplification in tongue and cheek squamous cell carcinoma. Asian Pac J Cancer Prev 2011;12:2199-204

23. Feng Z, Guo W, Zhang C, et al. CCND1 as a predictive biomarker of neoadjuvant chemotherapy in patients with locally advanced head and neck squamous cell carcinoma. PLoS One 2011;6:e26399

24. van Diest PJ, Michalides RJ, Jannink L, et al. Cyclin D1 expression in invasive breast cancer. Correlations and prognostic value. Am J Pathol 1997;150:705-11

25. Stransky N, Egloff AM, Tward AD, et al. The mutational landscape of head and neck squamous cell carcinoma. Science 2011;333:1157-60

26. Chung $\mathrm{CH}$, Zhang Q, Hammond EM, et al. Integrating epidermal growth factor receptor assay with clinical parameters improves risk classification for relapse and survival in head-and-neck squamous cell carcinoma. Int J Radiat Oncol Biol Phys 2011;81:331-38

27. Smith BD, Smith GL, Carter D, et al. Prognostic significance of vascular endothelial growth factor protein levels in oral and oropharyngeal squamous cell carcinoma. J Clin Oncol 2000;18:2046-52 